\title{
Research on Translation Based on Semantic Triangle Theory
}

\author{
Xiao Junming
}

Qiqihar Medical University, Qiqihar, Hei Longjiang, China, 161006

junming@163.com

Keywords: semantic triangle; translation; context

\begin{abstract}
Semantic triangle theory is one of the important theories of linguistics. This paper begins with a brief introduction to the connotation of semantic triangle theory, and then analyzes the concrete thinking process of translators in the translation process and the reading process of readers, finally gives the points for attention of English translation based on the semantic triangle theory in order to provide some references for the related researchers.
\end{abstract}

\section{Introduction to Semantic Triangle Theory}

Translation is an intercultural communication activity of human beings. A famous linguist named Armstrong Richards once said that translation is likely to be the most complex of human activities in the process of the evolution of the universe. The complexity of translation requires effective research in different ways. Linguistics as a perspective and method to translation studies, translation studies made great contribution, from Nida, Catford, Newmark, Wilss et al. They pay attention to the language of the common, emphasizing the consistency of the rules of language and the objective world, to seek the semantic equivalence and language conversion rules. In the author's view, the theory of "semantic triangle" in semantics is of positive significance to translation teaching and research. "Semantic triangle": the relationship between the three persons of "form and sound of the language sign". The semantic triangle in the book the Meaning of Meaning written by C.K.Ogden and I.A.Richards can be described as the following Figure 1:

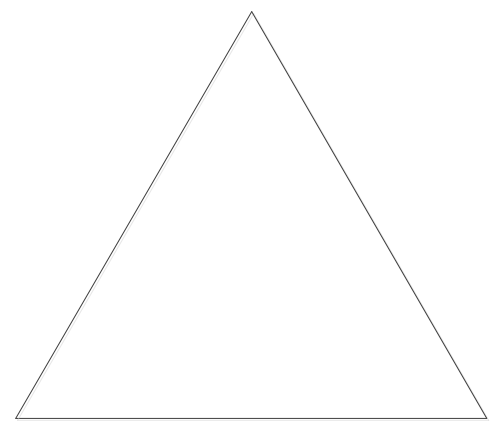

Figure 1Connotation of Semantic Triangle

C. K. Ogden and I.A.Richards clear defined the meaning and the object, through the symbol, which is "shape/sound and referent between the convention relationships to reflect mind to express the meaning of reference. In this "semantic triangle", "object" refers to the real existence of things in the objective world. For example, we see a tree, see is a real object. And at the same time our minds will appear the "meaning" of the "tree", is "semantic triangle" in "righteousness"; and if we want to tell others what we see "objects", "tree", we need to through sign language "form (tree) / tone " to express. That is to say, whether in written or verbal communication, we are referring to the "thing", by "thing" to form the "righteousness" is to express through "form/voice". In the literary works, the order in which the three are formed in the mind of the creator should be "the thing"is the" object "and" righteousness ". In the process of readers' understanding of literary works, the order should be meaning. Therefore, if the "form and sound" is not translated correctly, the "meaning" and "object" transmitted by it cannot be accurately reflected. Translation in this way 
cannot achieve the basic "faithfulness" in the three principles of translation, not to mention the "expressiveness" and "elegance". That is to say, the key to translation is through twosemiotic system of language formreasonable conversion and correctly reflect the original author described in the works "'" really want to express the "righteousness". We know that the internal symbol refers to the relationship between ("shape/tone") and signified ("meaning") between the relationship and symbol and "objects" are conventional. In theory, as long as there is a "thing", whether it is concrete or abstract, it should be a corresponding "meaning" to explain it ought to have corresponding "shape/tone" as a description of the interpretation of the vector. The specific things are easier to explain, and abstract things need to be explained in detail. For example: the abstract concept of loneliness needs to be explained by a specific thing, a lonely person. And in literary works, in order to heighten the theme, the author will also tend to use some can give people the "lonely" scenery to reflect it, such as: "independent old tree", "the night solitary" and so on. So the translator in the translation, besides should closely linked to the theme of the macroscopically "righteousness" outside, should also pay attention to every "shape/tone" whether can truly reflect the original in every individual "objects" micro "righteousness", because each a microscopic "righteousness" as the theme of the macro "righteousness" service. So in the translation we can't ignore any language symbols for symbolic language to convey the "righteousness". We should reproduce the original meaning, do reasonable extension and don't exaggerate any things.

\section{Semantic Triangle, Translator and Reader}

\section{Relationship between Semantic Triangle and Translator}

The translator's action is to translate a kind of language to another kind of language. From the view of the process of the semantic triangle theory, the translator first converts the symbolic meaning of the source language to the corresponding objects, and then by the thought of the translator into another language. This process can be described as Figure 2. This is represented by the semantic triangle theory translation translators should experience the process of thinking, the vast most translators is by the symbol 'a', after the translator's thinking directly to the symbol B, not after the object in the process. If symbol 'a' refers to object and the symbol B refers to the object, or a symbol refers to objects in another symbol resides in a culture does not exist, how to do? In the process of foreign language learning, learners tend to have a "fixed equivalents" concept, that the Chinese have then foreign language will also have a; any Chinese said a place; foreign language said a, and so on. A lot of people put aside the text of a special effort to memorize words to reflect this idea, so that the memory of the equivalent of the word, in fact, will become a barrier to understanding and using foreign language. For example, the word "Fenmu" is generally translated into grave or tomb. However, these two objects can only be regarded as similar, but not equal. Sometimes, the difference will lead to misunderstanding.

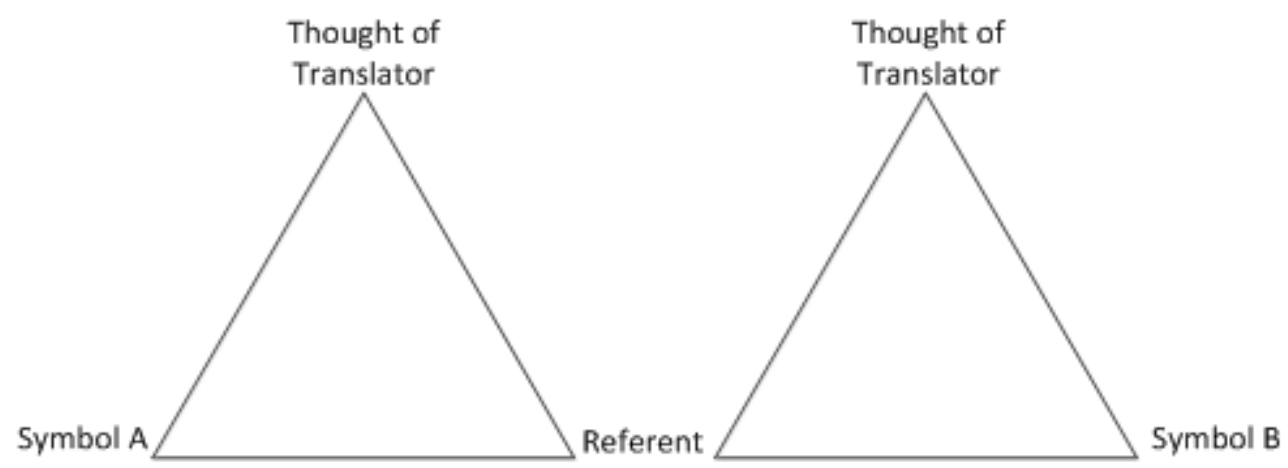

Figure 2 Process of Translation Based on Semantic Triangle Theory

\section{Relationship between Semantic Triangle and Reader}

Under normal circumstances, the author writes with an imaginary audience in his mind. He does not think of specific acceptance of personal characteristics of objects, but for this population 
common historical background, cultural characteristics, thinking and understanding, language habits are aware of. This commonality is good, and the effect is good. Clearly, the translator must also consider the expected effect of the target text with such a group. However, the source language readers and the target readers' historical background, cultural characteristics, thinking and language habits are not the same, for the same thing and even have the opposite understanding. A prominent example is the dragon". Chinese "dragon" is the embodiment of auspicious, noble, no wings, but can into the water and fly and fly. However, the western "dragon" is the embodiment of evil. The objects represented by the two are different and they are not the same. So if the Chinese "dragon" only translated into dragon is clearly not enough. As a translator, in the choice to translate the text should the reader in a specific group of people as their own imaginary recipients to the reader the common background, culture, habits of thought into account. Readers cannot continue to read an uninteresting and unreasonable article, still less to accept the views expressed in the article. Even if the selected text, the translator should always be from the perspective of the target readers to translate works. Because the readers in reading first see is a text symbol, then after the brain thinking, intentionally or unintentionally, the text symbols into objects in the world of experience and in the particular way of thinking to understand links between things, and events. Therefore, when the source text readers and the target text readers understand the same thing in different ways, the misunderstanding will be produced.

\section{Translation Based on Semantic Triangle}

\section{Selection between Original Meaning and Extended Meaning}

The lexical meaning of word often contains original meaning and extended meaning. When an English word translated into Chinese, it can be translated into a number of Chinese words. When choosing which one needs to understand the language knowledge including language culture, we should consider the original meaning and extended meaning. Semantic triangle theory is the basic theory that can effectively guide the translation practice. If $\mathrm{ABC}$ represents the source language, $\mathrm{ABC}$ represents the target language, and the translation is the process of $\mathrm{B}$ to $\mathrm{B}$. The equivalence in the target language is found by the same concept $\mathrm{A}$. For example, translators by B know primitives to a-c can in turn to find in the target language can refer to $\mathrm{B}$, thus completing the translation of a concept. In sentence translation, often fail to follow the original one to one meaning in translation. Because a single word appears in the sentence, it can also produce a relevant extension. Like "thirsty"is a word that has the same basic concept, it is different in different language system and culture, which is the manifestation of cultural differences. At this moment, it is necessary to define the meaning of the grammatical function and meaning of the corresponding language system in the "thirst". Another example is the "dog", the dog and the dog Chinese and no different, are four legs two ears can wag its tail, but in the subjective emotional but there is a significant difference. Americans like dogs, and think that it is a lucky dog and every dog has its day. Chinese not only eat dog meat, but also think the dog is inferior creatures, such as "worse than dogs and pigs", "running dogs" are very vicious curse words. We only have a full understanding of the significance of A and a are the reflection of $\mathrm{C}$ and c, we can have a good command of $\mathrm{B}$ and $\mathrm{b}$.

\section{Discretion of Chinese Culture and English Culture}

A and a often reflect thenatural environment and social environment. Proverbs, as a conventional language in the translation should pay attention to analysis of the language of the formation process. $\mathrm{B}$ and $\mathrm{b}$ reflect the real existence of $\mathrm{C}$ and $\mathrm{C}$ reflects through $\mathrm{A}$ and $\mathrm{a}$. The same can be used to analyze the semantic triangle, and then in the target language can be found in the translation of the reader's resonance. There is a saying: after drop fills the tub Drop. Drop refers to the of water Drop is small as a drop of water, but a drop of water droplets into the bucket, will be filled with a bucket, said that the small things cannot be ignored. Correspondingly, Chinese is one of the main expression of small things thought also has similar proverb "Dripping water wears through a stone". In fact with the English proverbs dripping water wears through rock based on the reference to the concept is exactly the same. Therefore, conversion from A-A can achieve the equivalent effect. On the other hand, in the understanding of abstract concepts, people with different languages and 
cultures have different processes. And make the same concept is expressed in different ways in different places. At this time, the translation is not at the word level, but on the sentence level. That is to say there is no way in the word level of equivalence translation. Walk on eggs, that is very careful to walk like, extended to the cautious movements and breathtaking, we Chinese people may sometimes do not understand why "walking on eggs", but an explanation can really very easy to accept, because our acrobatic or Kung Fu in the Dodge egg standing staged dodge. But we Chinese people are accustomed to "walking on thin ice" describe a person doing things very carefully and thrilling state, walk on the thin ice, it is necessary to be careful and always fall risk. The translation"on thin ice" should be called equivalent translation, also can be considered as a macro semantic triangle.

\section{Conclusion}

The purpose of the translation is to convey information to the target readers, so the translator should pay close attention to the reader's response in the process of translation. In terms of the words that may cause the reader to misunderstand, the translator should pay attention to select the original meaning or extended meaning and the difference between Chinese culture and English culture. Based on the semantic triangletheory, the translators can provide the target article with "faithfulness", "expressiveness" and "elegance”.

\section{References}

[1] Liang Hongping, Cross-culture Equivalence of TranslationUnderlying Semantic Triangle Theory, Journal of Zhanjiang Ocean University, 2004(5):80-83.

[2] Peng Liyuan, Xiao Yuetian, Rectifying the Classic Semantic Triangle, J., Foreign Language Research, 2009(4):51-54.

[3] Feng Anhua, On Translation of Object Names Based on Semantic Triangle Theory, J., Journal of Nantong Textile Vocational Technology College, 2013(4): 41-44.

[4] TanHuiying, A Comparison on theMing-Shi Idea of Pre-Chin and AncientGreek fromthe Point of View ofModern Semantic Triangle Theory, J., Foreign Language Research, 2011(2):9-15.

[5] Liu Yingpin, The Relationship between the Semantic Triangle Theory and the Principlesand Methods for English Lexical Teaching, J., Journal of Shenyang Agricultural University(Social Sciences Edition), 2014(5):326-329. 